ESAIM: PROCEEDINGS, April 2012, Vol. 36, p. 159-169

Danièle Fournier-Prunaret, Laura Gardini, \& Ludwig Reich, Editors

\title{
SUBSTITUTION SYSTEMS ASSOCIATED WITH THE DYNAMICAL SYSTEM $\left(\mathcal{A}, T_{f}\right)^{*}$
}

\author{
Maria de Fátima Correia ${ }^{1}$, Carlos Ramos ${ }^{1}$ and SAndra Vinagre ${ }^{1}$
}

\begin{abstract}
We consider the dynamical system $\left(\mathcal{A}, T_{f}\right)$, where $\mathcal{A}$ is a class of differential real functions defined on some interval and $T_{f}: \mathcal{A} \rightarrow \mathcal{A}$ is an operator $T_{f} \phi:=f \circ \phi$, where $f$ is a differentiable $m$-modal map. If we consider functions in $\mathcal{A}$ whose critical values are periodic points for $f$ then, we show how to define and characterize a substitution system associated with $\left(\mathcal{A}, T_{f}\right)$. For these substitution systems, we compute the growth rate of the new critical points, and observe that this growth is independent of the initial conditions.
\end{abstract}

AMS (2000) subject classification. Primary: 37B10, 39B12; Secondary: 68Q42, 26A18.

Keywords. Infinite dimensional dynamical systems, Iteration theory, Symbolic dynamics, Substitution systems.

Résumé. Nous considérons le système dynamique $\left(\mathcal{A}, T_{f}\right)$, où $\mathcal{A}$ est la classe des fonctions réelles différentielles définies dans un intervalle et $T_{f}: \mathcal{A} \rightarrow \mathcal{A}$ est un opérateur $T_{f} \phi:=f \circ \phi$, où $f$ est une application $m$-modale différentielle. Si nous considérons les fonctions de $\mathcal{A}$ dont les valeurs critiques sont les points périodiques de $f$ alors nous montrons comment définir et charactériser le système de substitution associé au $\left(\mathcal{A}, T_{f}\right)$. Pour ce système de substitution, nous calculons le taux de croissance des nouveaux points critiques, et nous observons que cette croissance est indépendante des conditions initiales.

Mots clefs. Systèmes dynamiques de dimension infinie, Théorie de l'itération, Dynamique symbolique, Système de substitution.

\section{INTRODUCTION}

In this paper, we pursue the study of the properties of certain infinite dynamical systems. Considering infinite dimensional systems, important progresses were obtained in the study of boundary value problems for partial differential equations reducible to difference equations, as those studied in [7], [11] and [12]. An approach, in that context, using symbolic dynamics was made in $[9,10]$ and $[13]$.

We deal with a certain type of infinite dimensional dynamical systems which arises from iterated diffenciable interval maps. We consider the symbolic dynamics of the functions in $\mathcal{A}$ (defined bellow) under iteration of a $m$-modal map developed in our previous work [3], as a consequence of the results obtained in [1,2], where we analysed a combinatorial description for the periodic orbits of $\left(\mathcal{A}, T_{f}\right)$. The pair $\left(\mathcal{A}, T_{f}\right)$ is the dynamical system we consider, where $\mathcal{A}$ is a class of differential real functions defined on some interval and $T_{f}: \mathcal{A} \rightarrow \mathcal{A}$ is an

* Supported by Calouste Gulbenkian Foundation and partially supported by the research center CIMA-UE, FCT-Portugal funding program.

${ }^{1}$ Department of Mathematics, University of Évora, Rua Romão Ramalho, 59, 7000-671 Évora, Portugal; e-mail: mfac@uevora.pt ccr@uevora.pt \& smv@uevora.pt.

(C) EDP Sciences, SMAI 2012 
operator $T_{f} \phi:=f \circ \phi$, where $f$ is a differentiable $m$-modal map. We start with the class of differentiable functions, $\mathcal{A}$, defined on an interval (eventually could be defined on $\mathbb{R}$ ) such that we say that $\phi \in \mathcal{A}$ if $\phi \in C^{1}([0,1])$, satisfy the boundary conditions $\phi^{\prime}(0)=\phi^{\prime}(1)=0$ and $\operatorname{Im}(\phi) \subset I$, for a given interval $I$. The nature of this class $\mathcal{A}$ in terms of topological, metrical or algebraic closure, for now, is not discussed. The former dynamical system which arises from iterated differentiable interval maps has infinite dimension, although induced by a one-dimensional discrete dynamical system $(I, f)$. From the topological point of view, the dynamical system $(I, f)$ is formally contained in $\left(\mathcal{A}, T_{f}\right)$, since the constant functions, $\phi \equiv c$, belong to $\mathcal{A}$ and $T_{f}(c)=f(c)$. Moreover, a monotone function $\phi \in \mathcal{A}$ (nontrivial) determine a signed interval, $([\phi(0), \phi(1)],+)$ if $\phi$ is an increasing function and $([\phi(1), \phi(0)],-)$ if $\phi$ is a decreasing function. Then the dynamics of intervals under iteration of an interval map $f$ is also formally contained in $\left(\mathcal{A}, T_{f}\right)$.

Symbolic substitution systems have had an important role in the study of dynamical systems. From a simple way, we can say that a susbstitution system is a pair composed by an alphabet and a map characterized by a set of rules which transform elements of a sequence into a new sequence.

In Section 3.2, we consider the subclass $\mathcal{A}_{\text {per }}$ of $\mathcal{A}$ which is constituted by functions $\phi$ whose critical values are periodic points of the $m$-modal map $f$ and we consider $m$-modal maps with periodic kneading sequences. Considering the functions in the class $\mathcal{A}_{\text {per }}$ we build and characterize a substitution system associated with $\left(\mathcal{A}, T_{f}\right)$ using the periodic kneading sequences as new elements in an alphabet. In general, substitutions replace a letter by a word which produces sequences under iteration, however, in our case, we must consider substitutions that use rules acting on a pair of letters which produce, by iteration, new sequences. For these substitution systems, we compute the growth rate of the size of the words which in turn corresponds to the growth rate of the new critical points in the initial system. Moreover, we observe that this growth rate is independent on the initial conditions from the class $\mathcal{A}_{\text {per }}$.

Now, in what follows we describe some preliminaries on symbolic dynamics, in particular, aspects concerning to the $m$-modal maps on an interval $I$.

\section{SYMBOLIC DYNAMICS FOR $m$-MODAL MAPS}

Let $I \subset \mathbb{R}$ be an interval. A map $f: I \rightarrow I$ is called m-modal map if is in $C^{1}(I)$ and has $m$ critical points.

Let $c_{i}$, with $i=1,2, \ldots, m$, be the $m$ critical points of the $m$-modal map $f$ such that $c_{0}<c_{1}<\cdots<c_{m+1}$, where $c_{0}$ and $c_{m+1}$ represent the boundary points of the interval $I$. In these conditions, consider the partition of the interval $I$ into disjoint subsets

$$
I=I_{1} \cup I_{C_{1}} \cup I_{2} \cup I_{C_{2}} \cup \cdots \cup I_{C_{m}} \cup I_{m+1},
$$

where $I_{C_{i}}$ is the set $\left\{c_{i}\right\}, i=1,2, \ldots, m$, and $I_{i}, i=1,2, \ldots, m+1$, are given by

$$
I_{1}=\left[c_{0}, c_{1}\right), I_{2}=\left(c_{1}, c_{2}\right), \ldots, I_{m+1}=\left(c_{m}, c_{m+1}\right] .
$$

Each interval $I_{i}, i=1,2, \ldots, m+1$, is an interval of monotonicity of $f$. Next, to each point $x$ in interval $I_{i}, i=1,2, \ldots, m+1$, we assign the symbol $i, i=1,2, \ldots, m+1$, or $C_{i}, i=1,2, \ldots, m$, if the point $x$ is the critical point $c_{i}, i=1,2, \ldots, m$ of $f$. This assignment is called the address of $x$ and it is denoted by $a d(x)$. The address of the point $x, a d(x)$, is thus given by

$$
a d(x)=\left\{\begin{array}{lll}
i & \text { if } \quad x \in I_{i}, \quad i=1,2, \ldots, m+1 \\
C_{i} & \text { if } \quad x \in I_{C_{i}}, \quad i=1,2, \ldots, m
\end{array}\right.
$$

As usual, we get a correspondence between orbits of points and symbolic sequences of the alphabet set $\left\{1, C_{1}, 2, \ldots, m+1\right\}$, the itinerary of $x$ under $f$ is defined by

$$
i t_{f}(x):=a d(x) a d(f(x)) a d\left(f^{2}(x)\right) \cdots \in\left\{1, C_{1}, 2, \ldots, m+1\right\}^{\mathbb{N}} .
$$


The orbits, under $f$, of the critical points are of special importance, in particular, their itineraries. Following J. Milnor and W. Thurston in [6], for each critical point of $f$, the kneading sequence is given by $\mathcal{K}_{i}:=i t_{f}\left(f\left(c_{i}\right)\right), i=1,2, \ldots, m$, and the collection of symbolic sequences $\mathcal{K}_{f}:=\left(\mathcal{K}_{1}, \ldots, \mathcal{K}_{m}\right)$ is called the kneading invariant of $f$.

A symbolic sequence $\left(S_{k}\right)_{k \geq 1}$ in $\left\{1, C_{1}, 2, \ldots, m+1\right\}^{\mathbb{N}_{0}}$ is called admissible, with respect to $f$, if it occurs as an itinerary for some point $x$ in $I$. The set of all admissible sequences in $\left\{1, C_{1}, 2, \ldots, m+1\right\}^{\mathbb{N}_{0}}$ is denoted by $\Sigma_{f}$

In the sequence space $\Sigma_{f}$, we define the usual shift map $\sigma: \Sigma_{f} \rightarrow \Sigma_{f}$ by

$$
\sigma\left(P_{1} P_{2} P_{3} \ldots\right)=P_{2} P_{3} \ldots
$$

Moreover, the following relation with $f$ and the itinerary map is satisfied

$$
\sigma\left(i t_{f}(x)\right)=i t_{f}(f(x))
$$

Therefore, we obtain the symbolic system $\left(\Sigma_{f}, \sigma\right)$ associated with the discrete dynamical system $(I, f)$.

An admissible word is a finite sub-sequence occurring in an admissible sequence. The set of admissible words of size $k$ occurring in some sequence from $\Sigma_{f}$ is denoted by $\mathcal{W}_{k}=\mathcal{W}_{k}(f)$.

Consider the sign function $\operatorname{sgn}: \cup_{k=1}^{\infty} \mathcal{W}_{k} \rightarrow\{-1,0,+1\}$ defined by

$$
\operatorname{sgn}\left(S_{1} \ldots S_{k}\right)=\prod_{j=1}^{k} \operatorname{sgn}(j),
$$

with $S_{1} \ldots S_{k} \in \mathcal{W}_{k}, \operatorname{sgn}\left(C_{j}\right)=0$ and $\operatorname{sgn}(j)=+1$ or $\operatorname{sgn}(j)=-1$, according $f$ restricted to $I_{j}$ is increasing or decreasing, respectively.

The parity, with respect to $f$, of a given admissible word $S_{1} \ldots S_{k} \in \mathcal{W}_{k}$ is even if $\operatorname{sgn}\left(S_{1} \ldots S_{k}\right)=+1$ and odd if $\operatorname{sgn}\left(S_{1} \ldots S_{k}\right)=-1$. From the order relation $1 \prec C_{1} \prec 2 \prec \cdots \prec m+1$, inherited from the order of the intervals of the partition of the interval $I$, we introduce an order relation between symbolic sequences as follows: given any distinct sequences $\left(P_{k}\right)_{k \geq 1},\left(Q_{k}\right)_{k \geq 1} \in\left\{1, C_{1}, 2, \ldots, m+1\right\}^{\mathbb{N}_{0}}$, admitting that they have a common initial subsequence, $i . e$, there is a natural $r \geq 1$ such that $P_{1} \ldots P_{r}=Q_{1} \ldots Q_{r}$ and $P_{r+1} \neq Q_{r+1}$, we will say that $\left(P_{k}\right)_{k \geq 1} \prec\left(Q_{k}\right)_{k \geq 1}$ if and only if $P_{r+1} \prec Q_{r+1}$ and $\operatorname{sgn}\left(P_{1} \ldots Q_{r}\right)=+1$ or $Q_{r+1} \prec P_{r+1}$ and $\operatorname{sgn}\left(P_{1} \ldots P_{r}\right)=-1$.

\section{Symbolic Dynamics for the infinite Dynamical System $\left(\mathcal{A}, T_{f}\right)$}

Now, consider a $m$-modal map $f$ in the class $C^{1}(I)$, for a certain interval $I$, and the class of differentiable functions

$$
\mathcal{A}=\left\{\varphi \in C^{1}([0,1]): \varphi^{\prime}(0)=\varphi^{\prime}(1)=0 \text { and } \operatorname{Im}(\varphi) \subset I\right\} .
$$

Let $T_{f}$ be the operator

$$
\begin{aligned}
T_{f}: \mathcal{A} & \rightarrow \mathcal{A} \\
\varphi & \mapsto f \circ \varphi
\end{aligned}
$$

Note that this operator is well defined since $(f \circ \varphi)^{\prime}(0)=(f \circ \varphi)^{\prime}(1)=0$. Moreover, if $\phi \in \mathcal{A}$ and $\operatorname{Im}(\phi) \subset I$ then $\operatorname{Im}\left(T_{f}^{k} \phi\right) \subset I$ for every $k \in \mathbb{N}$. Therefore, we obtain a discrete dynamical system $\left(\mathcal{A}, T_{f}\right)$ in the sense that we have a set $\mathcal{A}$ (eventually with additional structure, a topology or a metric, for now not specified) and a self map $T_{f}$, which characterises the discrete time evolution.

In order to introduce a symbolic description for the discrete dynamical system $\left(\mathcal{A}, T_{f}\right)$, let us consider the decomposition of $\mathcal{A}$ into the following classes, as in $[2,3]$ : 
and

$$
\begin{aligned}
& \mathcal{A}_{c}=\{\phi \in \mathcal{A}: \phi(x) \text { is constant in }[0,1]\}, \\
& \mathcal{A}_{0}=\{\phi \in \mathcal{A}: \phi \text { has no critical points in }(0,1)\}
\end{aligned}
$$

$$
\mathcal{A}_{j}=\{\phi \in \mathcal{A}: \phi \text { has } j \text { critical points in }(0,1)\}, j=1,2, \ldots
$$

Let $\phi \in \mathcal{A}$ and let $\eta(\phi)$ be the number of non-trivial critical points of $\phi$ (inside $[0,1])$. In this case if $\phi \in \mathcal{A}_{j}$, $j \in \mathbb{N}_{0}$, then $\eta(\phi)=j$ and the total number of critical points is $\eta(\phi)+2=j+2$. We are interested in the symbolic description of the dynamical evolution of a function $\phi$ under iteration of $f$. Moreover, we are interested that this symbolic description has essentially a topological meaning, therefore the important point is to distinguish and codify the critical points and values of $\phi$. Given $\phi \in \mathcal{A}$, we identify its critical points and collect the addresses and itineraries of the corresponding critical values. Our generalized symbolic space will be $\underline{\Sigma}_{f}:=\cup_{j \in \mathbb{N}_{0}} \Sigma_{f}^{j+1}$, where $\Sigma_{f}^{j+1}=\Sigma_{f} \times \Sigma_{f} \times \cdots \times \Sigma_{f}(j+1$ times $)$. In [3], we defined the generalized address, itinerary and shift maps for the space $\mathcal{A}$,

$$
\begin{aligned}
\underline{a d}: \mathcal{A} & \rightarrow \underline{\Sigma}_{f} \\
\phi & \mapsto \underline{a d}(\phi):=\left(\operatorname{ad}\left(d_{0}\right), \operatorname{ad}\left(d_{1}\right), \ldots, a d\left(d_{\eta(\phi)}\right), \operatorname{ad}\left(d_{\eta(\phi)+1}\right)\right), \\
\underline{i t}_{f}: \mathcal{A} & \rightarrow \underline{\Sigma}_{f} \\
\phi & \mapsto \underline{i t}_{f}(\phi)=\left(i t_{f}\left(d_{0}\right), i t_{f}\left(d_{1}\right), \ldots, i t_{f}\left(d_{\eta(\phi)}\right), i t_{f}\left(d_{\eta(\phi)+1}\right)\right),
\end{aligned}
$$

with $d_{j}:=\phi\left(a_{j}\right), j=1, \ldots \eta(\phi)$, where $a_{j}, j=1, \ldots, \eta(\phi)$, are the non-trivial critical points of $\phi$ in the interval $[0,1]$ (with $d_{0}=\phi(0)$ and $\left.d_{\eta(\phi)+1}=\phi(1)\right)$.

Let $S^{(j)}=S_{1}^{(j)} S_{2}^{(j)} \ldots, j=0,1,2, \ldots, \eta(\phi)+1$ such that $S^{(j)}=i t_{f}\left(d_{j}\right)$, where $d_{j}, j=0,1,2, \ldots, \eta(\phi)+1$, are the critical values of $\phi$. The extended shift map is then defined by

$$
\begin{aligned}
& \underline{\sigma}\left(S^{(0)}, \ldots, S^{(j)}, S^{(j+1)}, \ldots, S^{(\eta(\phi)+1)}\right):= \\
& =\left\{\begin{array}{c}
\left(\sigma\left(S^{(0)}\right), \ldots, \sigma\left(S^{(j)}\right), \sigma\left(S^{(j+1)}\right), \ldots, \sigma\left(S^{(\eta(\phi)+1)}\right)\right) \\
\text { if } S_{1}^{(j)}=S_{1}^{(j+1)}, \\
\left(\sigma\left(S^{(0)}\right), \ldots, \sigma\left(S^{(j)}\right), \mathcal{K}_{\left.S_{1}^{(j)}, \ldots, \mathcal{K}_{S_{1}^{(j+1)}-1}, \sigma\left(S^{(j+1)}\right), \ldots, \sigma\left(S^{(\eta(\phi)+1)}\right)\right)} \text { if } S_{1}^{(j)} \neq S_{1}^{(j+1)} \text { and } S_{1}^{(j)} \prec S_{1}^{(j+1)},\right. \\
\left(\sigma\left(S^{(0)}\right), \ldots, \sigma\left(S^{(j)}\right), \mathcal{K}_{S_{1}^{(j)}-1}, \ldots, \mathcal{K}_{\left.S_{1}^{(j+1)}, \sigma\left(S^{(j+1)}\right), \ldots, \sigma\left(S^{(\eta(\phi)+1)}\right)\right)}\right. \\
\text { if } S_{1}^{(j)} \neq S_{1}^{(j+1)} \text { and } S_{1}^{(j+1)} \prec S_{1}^{(j)},
\end{array}\right.
\end{aligned}
$$

where $\mathcal{K}_{S_{1}^{(j)}}$ with $S_{1}^{(j)} \in\{1, \ldots, m\}$ is the kneading sequence corresponding to the critical point of $f, c_{S_{1}^{(j)}}$, localized between $d_{j}$ and $d_{j+1}, j=0,1,2, \ldots, \eta(\phi)$.

Moreover, we obtained a symbolic system $\left(\underline{\Sigma}_{f}, \underline{\sigma}\right)$ associated to $\left(\mathcal{A}, T_{f}\right)$. Similarly to the finite dimensional discrete dynamical systems we obtained the following result, (see [2]):

Theorem 1. Let $\phi, \tilde{\phi} \in \mathcal{A}$ with $\phi \neq \tilde{\phi}$ so that $\underline{i t}_{f}(\phi)=\underline{i t}_{f}(\tilde{\phi})$, then

$$
\underline{i t}_{f}\left(T_{f}^{k} \phi\right)=\underline{i t}_{f}\left(T_{f}^{k} \tilde{\phi}\right), k \in \mathbb{N}_{0} .
$$


Moreover,

$$
\underline{\sigma} \circ \underline{i t}_{f}=\underline{i t}_{f} \circ T_{f}
$$

Next, we give an example to illustrate the previous definitions and results.

Example 1. Let us consider a bimodal map $f:[-1,1] \rightarrow[-1,1]$ which has the kneading invariant given by $\mathcal{K}_{f}=\left(\mathcal{K}_{1}, \mathcal{K}_{2}\right)$, where $\mathcal{K}_{1}=\left(332 C_{1}\right)^{\infty}$ and $\mathcal{K}_{2}=\left(112 C_{2}\right)^{\infty}$, with the alphabet $\left\{1, C_{1}, 2, C_{2}, 3\right\}$, it is given approximately by the analytical expression $f(x)=3.96754 x^{3}-2.96754 x$, see the Figure 1 . We consider in $\mathcal{A}$, an initial function such that $\underline{i t}_{f}(\phi)=\left((312)^{\infty},(32)^{\infty}\right)$.

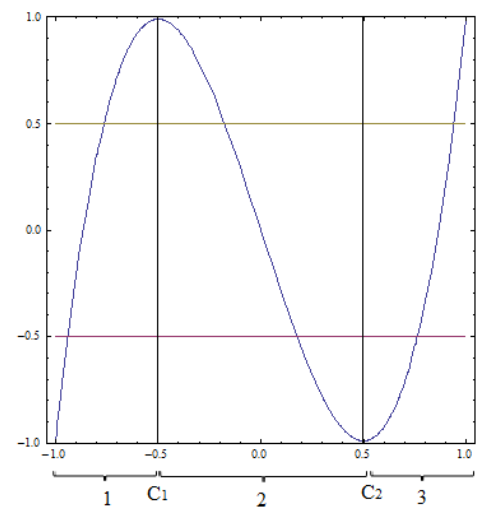

FIGURE 1. Graph of the bimodal map which analytical expression is given approximately by $f(x)=3.96754 x^{3}-2.96754 x$. The symbols of the alphabet $\left\{1, C_{1}, 2, C_{2}, 3\right\}$ are laid out horizontally in the legend at the bottom of the graph.

We have

$$
\underline{i t}_{f}(\phi)=\left((312)^{\infty},(32)^{\infty}\right) \in \Sigma_{f}^{2},
$$

then

$$
\begin{aligned}
\left.\underline{\sigma} \underline{i t}_{f}(\phi)\right)= & \left((123)^{\infty},(23)^{\infty}\right) \in \Sigma_{f}^{2}, \\
\left.\underline{\sigma}^{2} \underline{i t}_{f}(\phi)\right)= & \left((231)^{\infty}, \mathcal{K}_{1},(32)^{\infty}\right)= \\
= & \left((231)^{\infty},\left(332 C_{1}\right)^{\infty},(32)^{\infty}\right) \in \Sigma_{f}^{3}, \\
\left.\underline{\sigma}^{3} \underline{i t}_{f}(\phi)\right)= & \left((312)^{\infty}, \mathcal{K}_{2},\left(32 C_{1} 3\right)^{\infty},(23)^{\infty}\right)= \\
= & \left((312)^{\infty},\left(112 C_{2}\right)^{\infty},\left(32 C_{1} 3\right)^{\infty},(23)^{\infty}\right) \in \Sigma_{f}^{4}, \\
\underline{\sigma}^{4}\left(\underline{i t}_{f}(\phi)\right)= & \left((123)^{\infty}, \mathcal{K}_{2}, \mathcal{K}_{1},\left(12 C_{2} 1\right)^{\infty}, \mathcal{K}_{1}, \mathcal{K}_{2},\left(2 C_{1} 33\right)^{\infty}, \mathcal{K}_{2},(32)^{\infty}\right)= \\
= & \left((123)^{\infty},\left(112 C_{2}\right)^{\infty},\left(332 C_{1}\right)^{\infty},\left(12 C_{2} 1\right)^{\infty},\left(332 C_{1}\right)^{\infty},\left(112 C_{2}\right)^{\infty},\left(2 C_{1} 33\right)^{\infty},\left(112 C_{2}\right)^{\infty},(32)^{\infty}\right) \in \Sigma_{f}^{9}, \\
\underline{\sigma}^{5}\left(\underline{i t}_{f}(\phi)\right)= & \left((231)^{\infty},\left(12 C_{2} 1\right)^{\infty}, \mathcal{K}_{1}, \mathcal{K}_{2},\left(32 C_{1} 3\right)^{\infty}, \mathcal{K}_{2}, \mathcal{K}_{1},\left(2 C_{2} 11\right)^{\infty}, \mathcal{K}_{1}, \mathcal{K}_{2},\left(32 C_{1} 3\right)^{\infty}, \mathcal{K}_{2}, \mathcal{K}_{1},\left(12 C_{2} 1\right)^{\infty}, \mathcal{K}_{1},\right. \\
& \left.\left(C_{1} 332\right)^{\infty}, \mathcal{K}_{1},\left(12 C_{2} 1\right)^{\infty}, \mathcal{K}_{1}, \mathcal{K}_{2},(23)^{\infty}\right)= \\
= & \left((231)^{\infty},\left(12 C_{2} 1\right)^{\infty},\left(332 C_{1}\right)^{\infty},\left(112 C_{2}\right)^{\infty},\left(32 C_{1} 3\right)^{\infty},\left(112 C_{2}\right)^{\infty},\left(332 C_{1}\right)^{\infty},\left(2 C_{2} 11\right)^{\infty},\left(332 C_{1}\right)^{\infty},\right. \\
& \left(112 C_{2}\right)^{\infty},\left(32 C_{1} 3\right)^{\infty},\left(112 C_{2}\right)^{\infty},\left(332 C_{1}\right)^{\infty},\left(12 C_{2} 1\right)^{\infty},\left(332 C_{1}\right)^{\infty},\left(C_{1} 332\right)^{\infty},\left(332 C_{1}\right)^{\infty},
\end{aligned}
$$


and

$$
\begin{aligned}
\underline{i t}_{f}\left(T_{f} \phi\right)= & \left((123)^{\infty},(23)^{\infty}\right), \\
\underline{i t}_{f}\left(T_{f}^{2} \phi\right)= & \left((231)^{\infty}, \mathcal{K}_{1},(32)^{\infty}\right), \\
\underline{i t}_{f}\left(T_{f}^{3} \phi\right)= & \left((312)^{\infty}, \mathcal{K}_{2},\left(32 C_{1} 3\right)^{\infty},(23)^{\infty}\right), \\
\underline{i t}_{f}\left(T_{f}^{4} \phi\right)= & \left((123)^{\infty}, \mathcal{K}_{2}, \mathcal{K}_{1},\left(12 C_{2} 1\right)^{\infty}, \mathcal{K}_{1}, \mathcal{K}_{2},\left(2 C_{1} 33\right)^{\infty}, \mathcal{K}_{2},(32)^{\infty}\right), \\
\underline{i t}_{f}\left(T_{f}^{5} \phi\right)= & \left((231)^{\infty},\left(12 C_{2} 1\right)^{\infty}, \mathcal{K}_{1}, \mathcal{K}_{2},\left(32 C_{1} 3\right)^{\infty}, \mathcal{K}_{2}, \mathcal{K}_{1},\left(2 C_{2} 11\right)^{\infty}, \mathcal{K}_{1}, \mathcal{K}_{2},\left(32 C_{1} 3\right)^{\infty}, \mathcal{K}_{2}, \mathcal{K}_{1},\left(12 C_{2} 1\right)^{\infty}, \mathcal{K}_{1},\right. \\
& \left.\left(C_{1} 332\right)^{\infty}, \mathcal{K}_{1},\left(12 C_{2} 1\right)^{\infty}, \mathcal{K}_{1}, \mathcal{K}_{2},(23)^{\infty}\right) .
\end{aligned}
$$

We can verify that

$$
\underline{\sigma}^{k}\left(\underline{i t}_{f}(\phi)\right)=\underline{i t}_{f}\left(T_{f}^{k} \phi\right), \quad k=0,1,2, \ldots
$$

\section{Substitution systems}

\subsection{Basic facts about substitution systems}

Here we briefly recall some basic facts and terminology about substitutions systems, see [4]. By an alphabet we mean a finite nonempty set of symbols and denote by $\mathcal{S}$. We refer to the members of the alphabet $\mathcal{S}$ as letters. A word of length $k$ is an expression $s=s_{1} s_{2} \ldots s_{k}$, where each $s_{j}$ is a letter of $\mathcal{S}$. We write $|s|$ for the length of a word $s$. It is convenient to define the empty word $\epsilon$, having length 0 and satisfying the concatenation rule $\epsilon s=s=s \epsilon$ for every $s$.

Let $\mathcal{S}^{+}$be the set of all words of positive length in the alphabet $\mathcal{S}$ and set $\mathcal{S}^{*}=\mathcal{S}^{+} \cup\{\epsilon\}$.

We write $l_{s}(w)$ for the number of occurrences of the word $s$ in the word $w$. We say that a word $u \in \mathcal{S}^{+}$is a factor of a word $v \in \mathcal{S}^{+}$if $u=v_{m} v_{m+1} \ldots v_{n}$, with $v_{j} \in \mathcal{S}$ for some $0 \leq m \leq n<|v|$ and denote by $u=v_{[m, n]}$.

A substitution on the alphabet $\mathcal{S}$ is a $\operatorname{map} \zeta: \mathcal{S} \rightarrow \mathcal{S}^{*}$. The substitution map $\zeta$ is extended to the maps (also denoted by $\zeta$ ) $\zeta: \mathcal{S}^{*} \rightarrow \mathcal{S}^{*}, \zeta: \mathcal{S}^{+} \rightarrow \mathcal{S}^{+}$and $\zeta: \mathcal{S}^{\mathbb{N}} \rightarrow \mathcal{S}^{\mathbb{N}}$ by concatenation. Moreover, using the extention to words by concatenation, $\zeta$ can be iterated and $\zeta^{n}: \mathcal{S} \rightarrow \mathcal{S}^{*}$ is again a substitution.

The language of a sequence $u=\left(u_{n}\right)_{n \in \mathbb{N}} \in \mathcal{S}^{\mathbb{N}}$ is the set of all finite words that occur in $u$ and is denoted by $\mathcal{L}(u)$. The language of a substitution $\zeta$ is the set $\mathcal{L}(\zeta)$ of all factors of the words $\zeta^{n}(j)$, with $n \in \mathbb{N}$ and $j \in \mathcal{S}$.

The substitution system can be considered as the pair $(\mathcal{L}(\zeta), \zeta)$.

\subsection{Substitution systems associated with the symbolic dynamical system $\left(\mathcal{A}, T_{f}\right)$}

We consider the subclass $\mathcal{A}_{\text {per }}$ of $\mathcal{A}$ which is constituted by functions $\phi$ whose critical values are periodic points of the map $f$. Note that if $f$ is topological transitive then the periodic points of $f$ are dense in the interval $I$ and given $\tilde{\phi} \in \mathcal{A}$ we can choose $\phi \in \mathcal{A}_{\text {per }}$ arbitrarily close to $\tilde{\phi}$ (for a given metric).

Consider the kneading invariant of the map $f, \mathcal{K}_{f}=\left(\mathcal{K}_{1}, \ldots, \mathcal{K}_{m}\right)$, where $\mathcal{K}_{i}$ are periodic kneading sequences of period $p_{i}, i=1, \ldots, m$, and the set of the shifted sequences

$$
\bigcup_{i=1}^{m}\left\{\sigma^{l}\left(\mathcal{K}_{i}\right), l=0,1, \ldots, p_{i}-1\right\}
$$

Let us denote by $\mathcal{K}_{i}(l)$ the shifted kneading sequences $\sigma^{l}\left(\mathcal{K}_{i}\right), l=0,1, \ldots, p_{i}-1$.

Consider the correspondence between each shifted kneading sequence, $\mathcal{K}_{i}(l), i=1, \ldots, m, l=0,1, \ldots, p_{i}-1$, and a letter on the alphabet set $\mathcal{S}=\{1, \ldots, p\}, p=p_{1}+\cdots+p_{m}$, 


$$
\begin{aligned}
& \mathcal{K}_{1}(l) \rightarrow l+1, \quad l=0,1, \ldots, p_{1}-1, \\
& \mathcal{K}_{i}(l) \rightarrow p_{1}+\cdots+p_{i-1}+l+1, \quad l=0,1, \ldots, p_{i}-1, i=2, \ldots, m .
\end{aligned}
$$

Denote by 0 the empty word $\epsilon$.

Let $\mathcal{S}^{*}=\bigcup_{k=1}^{\infty}\{1, \ldots, p\}$ be the set of finite words of symbols from $\mathcal{S}$ (the letter 0 is included). Let us denote the set $\left\{1, p_{1}+1, p_{1}+p_{2}+1, \ldots, p_{1}+\cdots+p_{m-1}+1\right\}$ by $\mathcal{S}_{\mathcal{K}}$, which is the set of letters associated with the kneading sequences of the map $f$.

Given $\phi \in \mathcal{A}_{\text {per }}$, we consider also the alphabet set $\mathcal{S}(\phi)=\left\{A_{1}, \ldots, A_{t_{0}}, B_{1}, \ldots, B_{t_{1}}, \ldots\right\}$, where $A_{1}, B_{1}, \ldots$, are the letters associated with $i t\left(d_{0}\right), i t\left(d_{1}\right), \ldots$, the itineraries of the critical points of $\phi$ from (2) in Section 2, that is,

$$
\begin{array}{ll}
i t\left(d_{0}\right) & \rightarrow A_{1}, \\
i t\left(d_{1}\right) & \rightarrow B_{1},
\end{array}
$$

and

$$
\begin{array}{lll}
A_{j} \rightarrow \sigma^{j-1}\left(i t\left(d_{0}\right)\right), & & j=1,2, \ldots, t_{0}, \\
B_{j} \rightarrow \sigma^{j-1}\left(i t\left(d_{1}\right)\right), & j=1,2, \ldots, t_{1},
\end{array}
$$

By construction and from the previous definitions we have the result:

Theorem 2. Given a map $f$ with kneading invariant $\mathcal{K}_{f}=\left(\mathcal{K}_{1}, \ldots, \mathcal{K}_{m}\right)$ and $\phi \in \mathcal{A}_{\text {per }}$, consider the alphabet $\mathcal{S}=\{1, \ldots, p\}, p=p_{1}+\cdots+p_{m}$, with the correspondence (3), and $\mathcal{S}(\phi)=\left\{A_{1}, \ldots, A_{t_{0}}, B_{1}, \ldots, B_{t_{1}}, \ldots\right\}$, with the correspondences (4) and (5). Then, the generalized shift map on $\underline{i t}\left(\mathcal{A}_{\text {per }}\right)$, $\underline{\sigma}: \underline{i t}\left(\mathcal{A}_{\text {per }}\right) \rightarrow \underline{i t}\left(\mathcal{A}_{\text {per }}\right)$, induces a substitution system in the alphabet set $\mathcal{S} \cup \mathcal{S}(\phi)$ given by

$$
\begin{aligned}
& 1 \stackrel{\zeta}{\rightarrow} 2 \stackrel{\zeta}{\rightarrow} \ldots \stackrel{\zeta}{\rightarrow} p_{1} \stackrel{\zeta}{\rightarrow} 1, \\
& p_{1}+1 \stackrel{\zeta}{\rightarrow} p_{1}+2 \stackrel{\zeta}{\rightarrow} \ldots \stackrel{\zeta}{\rightarrow} p_{1}+p_{2} \stackrel{\zeta}{\rightarrow} p_{1}+1 \\
& \vdots \\
& p_{1}+\cdots+p_{m-1}+1 \stackrel{\zeta}{\rightarrow} p_{1}+\cdots+p_{m-1}+2 \stackrel{\zeta}{\rightarrow} \ldots \stackrel{\zeta}{\rightarrow} p_{1}+\cdots+p_{m} \stackrel{\zeta}{\rightarrow} p_{1}+\cdots+p_{m-1}+1 \text {, } \\
& A_{1} \stackrel{\zeta}{\rightarrow} A_{2} \stackrel{\zeta}{\rightarrow} \ldots \stackrel{\zeta}{\rightarrow} A_{t_{0}} \stackrel{\zeta}{\rightarrow} A_{1} \\
& B_{1} \stackrel{\zeta}{\rightarrow} B_{2} \stackrel{\zeta}{\rightarrow} \ldots \stackrel{\zeta}{\rightarrow} B_{t_{1}} \stackrel{\zeta}{\rightarrow} B_{1}
\end{aligned}
$$

and

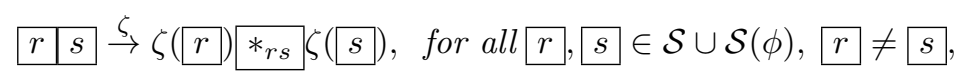

where $*_{r s}$ represents the letters in the set $\mathcal{L} \cup\{0\}$, associated with the kneading sequences which are between the first symbols of the sequences associated with $r$ and $s$. Note that $*_{r s}$ can represent an empty word.

Proposition 1. Let $\zeta: \mathcal{L} \rightarrow \mathcal{L}$ be the substitution defined in (6) with the alphabet set $\mathcal{S}=\{1, \ldots, p\}$, $p=p_{1}+\cdots+p_{m}$. Then, the words of the form

$$
\begin{array}{|l|l|}
\hline r & r \\
\hline
\end{array}
$$


cannot occur as factor of any word in $\mathcal{L}$, for all $r \in \mathcal{S}$.

Proof. Let us suppose that the word $r \mid r$ occurs as a factor of some word in $\mathcal{L}$, i.e., there is a word in $\mathcal{S}^{*}$ such that $\zeta(w)=r|r| r$. The word $w$ has the form $w=q \mid s$, with $q \neq q$ such that $\zeta(|q| s)=r \mid r$ which implies $\zeta(\sqrt{q})=r$ and $\zeta(\sqrt{s})=r$ and thus, $q=s$. Since $r|r| r$ cannot be associated with a generalized address of any $\phi \in \mathcal{A}_{\text {per }}$, the claim follows.

In order to ilustrate the previous definitions and results, let us consider the following example.

Example 2. Let us consider the bimodal map $f:[-1,1] \rightarrow[-1,1]$ from the Example 1, which has periodic kneading sequences given by $\mathcal{K}_{1}=\left(332 C_{1}\right)^{\infty}$ and $\mathcal{K}_{2}=\left(112 C_{2}\right)^{\infty}$, with the alphabet $\left\{1, C_{1}, 2, C_{2}, 3\right\}$. The map $f$ is approximately given by $f(x)=3.96754 x^{3}-2.96754 x$. Let $\phi_{0} \in \mathcal{A}_{\text {per }}$ be the initial function such that $\underline{i t}_{f}\left(\phi_{0}\right)=\left((312)^{\infty},(32)^{\infty}\right)$. The shifted sequences of the itinerary of $\phi_{0}$ are substituted as following

$$
(312)^{\infty} \rightarrow A_{1},(123)^{\infty} \rightarrow A_{2},(231)^{\infty} \rightarrow A_{3},(32)^{\infty} \rightarrow B_{1},(23)^{\infty} \rightarrow B_{2},
$$

and the shifted kneading sequences are substitued as following

$$
\begin{aligned}
& \mathcal{K}_{1}(0)=\left(332 C_{1}\right)^{\infty} \rightarrow 1, \mathcal{K}_{1}(1)=\left(32 C_{1} 3\right)^{\infty} \rightarrow 2, \\
& \mathcal{K}_{2}(0)=\left(112 C_{2}\right)^{\infty} \rightarrow 5, \mathcal{K}_{1}(2)=\left(2 C_{1} 33\right)^{\infty} \rightarrow 3, \\
& \mathcal{K}_{2}(1)=\left(12 C_{2} 1\right)^{\infty} \rightarrow 6,
\end{aligned}
$$

In this case, we get the alphabets $\mathcal{S}=\{1,2, \ldots, 8\}, \mathcal{S}_{\mathcal{K}}=\{1,1,5\}$ and $\mathcal{S}\left(\phi_{0}\right)=\left\{A_{1}, A_{2}, A_{3}, B_{1}, B_{2}\right\}$.

The substitution map $\zeta: \mathcal{L}(\zeta) \rightarrow \mathcal{L}(\zeta)$ is defined in the following way,

$$
\begin{aligned}
& 1 \stackrel{\zeta}{\rightarrow} 2 \stackrel{\zeta}{\rightarrow} 3 \stackrel{\zeta}{\rightarrow} 4 \stackrel{\zeta}{\rightarrow} 1 \text {, } \\
& 5 \stackrel{\zeta}{\rightarrow} 6 \stackrel{\zeta}{\rightarrow} 7 \stackrel{\zeta}{\rightarrow} 8 \stackrel{\zeta}{\rightarrow} 5 \text {, }
\end{aligned}
$$

$$
\begin{aligned}
& \begin{array}{|l|l|l|l|l|}
\hline 1 & 2 & \stackrel{\zeta}{\rightarrow} & 2 & 3 \\
\cline { 1 - 2 } & &
\end{array}
\end{aligned}
$$

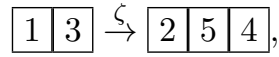

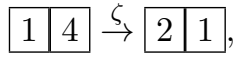

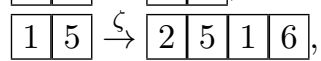

$$
\begin{aligned}
& \begin{array}{|l|l|l|l|l|l|}
\hline \hline 1 & 6 & \zeta \\
\hline & 2 & 5 & 1 & 7 \\
\hline
\end{array} \\
& \begin{array}{ll|l|l|l|l|}
\hline 1 & 7 & \zeta & \multicolumn{5}{l|}{} & 2 & 5 & 8 \\
\hline
\end{array} \\
& \begin{array}{ll|lll|l}
\hline 1 & 8 & \zeta & \multicolumn{1}{l|}{2} & 5 & 5,
\end{array} \\
& \begin{array}{|l|ll|l|}
\hline 2 & 1 & \zeta \\
& 3 & 2 & 2 \\
\hline
\end{array}
\end{aligned}
$$

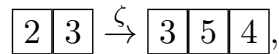

$$
\begin{aligned}
& \begin{array}{|l|l|l|l|}
\hline 2 & 4 & \zeta & \multicolumn{5}{|c}{3} & 1 & 1, \\
\hline
\end{array}
\end{aligned}
$$

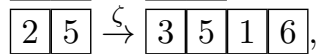

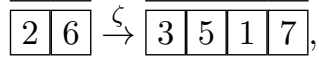

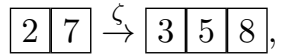

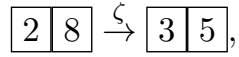

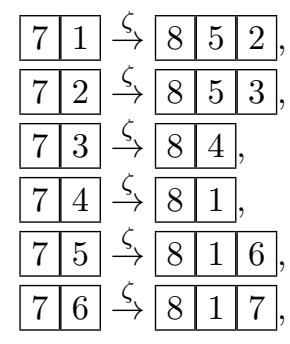

\begin{tabular}{|l|l|l|l|}
\hline 7 & 8 & \multicolumn{5}{c}{$\rightarrow$} & 8 & 5 \\
\hline
\end{tabular}

\begin{tabular}{|l|l|l|l|l|l|l|l|l|l|}
\hline 8 & 1 & \multicolumn{5}{c}{} & 5 & 2 & \\
\hline
\end{tabular}

\begin{tabular}{l|l|l|l|l|l|l|l|l|l|l|l|l|l|l|}
\hline 8 & 2 & \multicolumn{5}{c}{5} & 3 \\
\hline
\end{tabular}

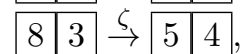

\begin{tabular}{|l|l|l|l|l|l|l|}
\hline 8 & 4 & $\zeta$ & 5 & 5 & 1 & \\
\hline
\end{tabular}

\begin{tabular}{|l|l|l|l|l|l|}
\hline 8 & 5 & $\zeta$ & 5 & 5 & 6 \\
\hline
\end{tabular}

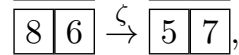

\begin{tabular}{|l|l|l|l|l|l|}
\hline 8 & 7 & \multicolumn{5}{c}{5} & 5 & 8 \\
\cline { 1 - 3 }
\end{tabular} 


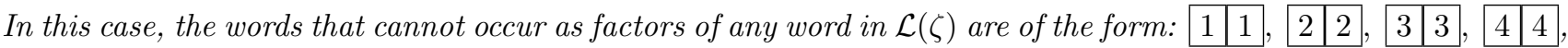

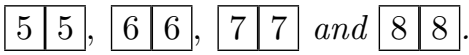

According to the itineraries of $\phi_{k}=f^{k}\left(\phi_{0}\right), k=1, \ldots, 5$, from the Example 1, and using (4)-(8), we obtained the following itineraries for $\phi_{k}=f^{k}\left(\phi_{0}\right), k=1, \ldots, 6$, in the alphabet $\mathcal{S}$. Each sequence corresponding to the itineraries is obtained by the iteration of the substitution in (9). The downward arrows indicate that the itineraries below are obtained through the substitution $\zeta$ from itineraries of the one above.

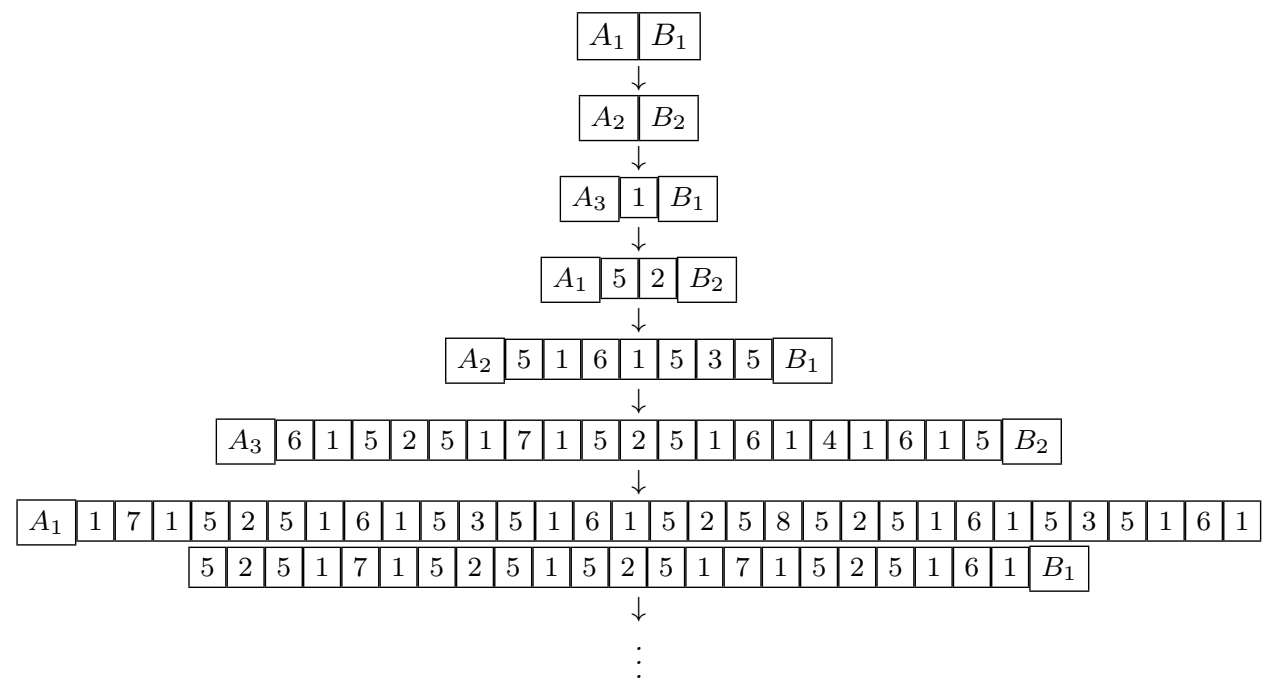

Example 3. Let us consider a bimodal map (from the Example 2) $f:[-1,1] \rightarrow[-1,1]$ which has the periodic kneading sequences $\mathcal{K}_{1}=\left(332 C_{1}\right)^{\infty}$ and $\mathcal{K}_{2}=\left(112 C_{2}\right)^{\infty}$, and the initial functions, in $\mathcal{A}_{\text {per }}$, whose itineraries are given by: $\underline{i t}_{f}\left(\phi_{01}\right)=\left((312)^{\infty},(32)^{\infty}\right), \underline{i t}_{f}\left(\phi_{02}\right)=\left((312)^{\infty},(121)^{\infty},(32)^{\infty}\right), \underline{i t}_{f}\left(\phi_{03}\right)=\left((123)^{\infty},(331)^{\infty},(112)^{\infty}\right)$ and $\underline{i t}_{f}\left(\phi_{04}\right)=\left((123)^{\infty},(331)^{\infty}\right)$.

Let $N_{k}$ be the number of the new critical values at each iteration $\phi_{k}, k=1,2, \ldots, 14$. The values of $\ln \left(N_{k}\right)$ and $\ln \left(N_{k+1} / N_{k}\right)$ corresponding to the initial functions $\phi_{01}, \phi_{02}, \phi_{03}$ and $\phi_{04}$, for each $k=1,2, \ldots$, are computed and displayed in the Table 1 and Table 2, respectively:

All the outputs of $\ln \left(\frac{N_{k+1}}{N_{k}}\right)$, from the Table 2 , increases with the values of $k=2, \ldots, 13$. Therefore, we have that

for $k=10,11, \ldots$

$$
\ln \left(\frac{N_{k+1}}{N_{k}}\right) \approx 1.018
$$

In the Figure 2, we present the variation of the number of the new critical values of function $\phi_{k}$, with iteration $k=1,2, \ldots, 12$, where the initial function has itinerary $\underline{i t}_{f}\left(\phi_{04}\right)=\left((123)^{\infty},(331)^{\infty}\right)$.

Example 4. Let us consider the bimodal maps $f:[-1,1] \rightarrow[-1,1]$ with periodic kneading sequences, $\mathcal{K}_{1}$ and $\mathcal{K}_{2}$, whose itineraries are listed in the Table 3. We consider the initial functions, in $\mathcal{A}_{\text {per }}$, whose itineraries are given by: $\underline{i t}_{f}\left(\phi_{01}\right)=\left((3222)^{\infty},(23)^{\infty}\right)$, $\underline{i t}_{f}\left(\phi_{02}\right)=\left((133)^{\infty},(223)^{\infty}\right)$ and $\underline{i t}_{f}\left(\phi_{03}\right)=\left((1323)^{\infty},(322)^{\infty}\right)$.

Let $N_{k}$ be the number of the new critical values at each iteration $\phi_{k}, k=1,2, \ldots, 19$. In the Table 3, we present the values for $\ln \left(N_{k+1} / N_{k}\right), k=10,12,16,17,18,19$, of the initial functions $\phi_{01}$, $\phi_{02}$ and $\phi_{03}$. For each bimodal map which respective kneading invariant, $\mathcal{K}_{f}$, is indicated in the first column from the Table 3 , we calculated the topological entropy of $f, h_{t o p}(f)$, through the transition matrix associated to each invariant $($ see $[5,8])$. 


\begin{tabular}{|c|c|c|c|c|}
\hline$k$ & $\phi_{01}$ & $\phi_{02}$ & $\phi_{03}$ & $\phi_{04}$ \\
\hline 2 & 0 & 2.48491 & 2.77259 & 1.94591 \\
\hline 3 & 0.693147 & 3.43399 & 3.80666 & 3.04452 \\
\hline 4 & 1.94591 & 4.38203 & 4.82831 & 4.07754 \\
\hline 5 & 2.94444 & 5.3845 & 5.84064 & 5.09987 \\
\hline 6 & 3.97029 & 6.40026 & 6.8533 & 6.11368 \\
\hline 7 & 4.97673 & 7.42058 & 7.87284 & 7.13409 \\
\hline 8 & 5.99645 & 8.43923 & 8.89151 & 8.15277 \\
\hline 9 & 7.01481 & 9.45767 & 9.91012 & 9.1715 \\
\hline 10 & 8.03398 & 10.4761 & 10.9287 & 10.1901 \\
\hline 11 & 9.05252 & 11.4946 & 11.9473 & 11.2087 \\
\hline 12 & 10.0711 & 12.5132 & & 12.2273 \\
\hline 13 & 11.0897 & & & \\
\hline 14 & 12.1083 & & & \\
\hline
\end{tabular}

TABLE 1. The first column indicates the number of the iteration $k$ and the rows indicate the $\ln \left(N_{k}\right), k=2, \ldots, 14$, of the $k^{t h}$-iterations under the map $f$ of $\phi_{01}, \phi_{02}, \phi_{03}$ and $\phi_{04}$. Note that the $k$ values start at 2 and increment by 1 until to 14 .

\begin{tabular}{|c|c|c|c|c|}
\hline$k$ & $\phi_{01}$ & $\phi_{02}$ & $\phi_{03}$ & $\phi_{04}$ \\
\hline 2 & 0.693147 & 0.85745 & 1.03407 & 1.09861 \\
\hline 3 & 1.25276 & 0.910212 & 1.02165 & 1.03302 \\
\hline 4 & 0.998529 & 0.986908 & 1.01233 & 1.02233 \\
\hline 5 & 1.02585 & 1.00995 & 1.01266 & 1.01382 \\
\hline 6 & 1.00644 & 1.0182 & 1.01954 & 1.02041 \\
\hline 7 & 1.01972 & 1.01789 & 1.01868 & 1.01868 \\
\hline 8 & 1.01836 & 1.01816 & 1.0186 & 1.01872 \\
\hline 9 & 1.01917 & 1.01831 & 1.01861 & 1.01864 \\
\hline 10 & 1.01853 & 1.01852 & 1.01858 & 1.0186 \\
\hline 11 & 1.0186 & 1.01857 & & 1.01858 \\
\hline 12 & 1.01856 & & & \\
\hline 13 & 1.01859 & & & \\
\hline
\end{tabular}

TABLE 2. The first column indicates the number of the iteration $k$ and the rows indicate the $\ln \left(N_{k+1} / N_{k}\right), k=2, \ldots, 14$, of the $k^{t h}$-iterations under the map $f$ of $\phi_{01}, \phi_{02}, \phi_{03}$ and $\phi_{04}$. Note that the $k$ values start at 2 and increment by 1 until to 13 .

According to the Table 3, we can observe that, for each bimodal map with positive topological entropy, $h_{\text {top }}(f)$, the growth rate of the new critical values for $\phi_{k}, k \in \mathbb{N}$, is independent to the initial conditions. Moreover, the growth rate of the new critical values for $\phi_{k}, k \in \mathbb{N}$, is approximately equal to the topological entropy of the bimodal map $f$. 


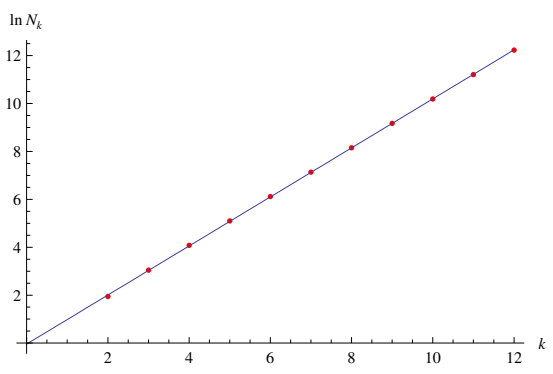

Figure 2. The variation of the number of the new critical values of $\phi_{k}$, with iterations $k=$ $1,2, \ldots, 12$, where the initial function has itinerary $\underline{i t}_{f}\left(\phi_{04}\right)=\left((123)^{\infty},(331)^{\infty}\right)$. The linear regression line with slope approximately is equal to 1.018 , fits the values of the last column of the Table 1.

\begin{tabular}{|c|c|c|c|c|}
\hline$\left(\mathcal{K}_{1}, \mathcal{K}_{2}\right)$ & $h_{\text {top }}(f)$ & $\phi_{01}$ & $\phi_{02}$ & $\phi_{03}$ \\
\hline$\left(\left(333 C_{1}\right)^{\infty},\left(111 C_{2}\right)^{\infty}\right)$ & 1.061260 & 1.06126 & 1.06136 & 1.06126 \\
\hline$\left(\left(332 C_{1}\right)^{\infty},\left(112 C_{2}\right)^{\infty}\right)$ & 1.018490 & 1.01859 & 1.0186 & 1.01857 \\
\hline$\left(\left(332 C_{1}\right)^{\infty},\left(132 C_{2}\right)^{\infty}\right)$ & 0.745265 & 0.744997 & 0.745628 & 0.745442 \\
\hline$\left(\left(333 C_{1}\right)^{\infty},\left(133 C_{2}\right)^{\infty}\right)$ & 0.609222 & 0.609371 & 0.609504 & 0.609504 \\
\hline
\end{tabular}

TABLE 3. After third column the rows indicate the $\ln \left(N_{k+1} / N_{k}\right), k=10,12,16,17,18,19$, of the $k^{t h}$-iterations under the map $f$ of $\phi_{01}, \phi_{02}$ and $\phi_{03}$.

\section{REFERENCES}

[1] M. F. Correia, C. C. Ramos and S. Vinagre, On the iteration of smooth maps, Discrete Dynamics and Difference Equations, Proceedings of the ICDEA'2007, World Scientific Publishing, 2010.

[2] M. F. Correia, C. C. Ramos and S. Vinagre, Symbolic dynamics for iterated smooth functions, Grazer Math. Berichte, 354, 26-36, 2009 .

[3] M. F. Correia, C. C. Ramos and S. Vinagre, Iteration of differentiable functions under m-modal maps, 2010 (submitted).

[4] F. Durand, F. Host, C. Skau, Substitution dynamical systems, Bratteli diagrams and dimension groups, Ergod. Th. \& Dynam. Sys., 19, 953-993, 1999.

[5] J. P. Lampreia and J. Sousa Ramos, Symbolic dynamics of bimodal maps, Port. Math. (N.S.), 54, Fasc.1, 1-18, 1997.

[6] J. Milnor and W. Thurston, On iterated maps of the interval in: J.C. Alexander(ed.) Proceedings Univ. Maryland, 1986-87, Lect. Notes in Math., 1342, Springer Verlag Berlin New York, 465-563, 1988.

[7] E. Yu. Romanenko and A. N. Sharkovsky, From boundary value problems to difference equations: a method of investigation of chaotic vibrations, Internat J. Bifur. Chaos Appl. Sci. Engrg. 9, 7, 1285-1306, 1999.

[8] R. Severino, Invariantes Topológicos e Algébricos em Sistemas Dinâmicos Discretos, PhD thesis, IST, 2000.

[9] R. Severino, A. N. Sharkovsky, J. Sousa Ramos and S. Vinagre, Symbolic Dynamics in Boundary Value problems, Grazer Math. Berichte, 346, 393-402, 2004.

[10] R. Severino, A. N. Sharkovsky, J. Sousa Ramos and S. Vinagre, Topological invariants in a model of a time-delayed Chua's circuit, Nonlinear Dynamics, 44, 81-90, 2004.

[11] A. N. Sharkovsky, Yu. Maistrenko and E. Yu. Romanenko, Difference equations and their applications, Kluwer Academic Publishers, 1993.

[12] A. N. Sharkovsky, Difference equations and boundary value problems, In "New Progress in Difference Equations", Proceedings of the ICDEA'2001, Taylor and Francis, 3-22, 2003.

[13] S. Vinagre, R. Severino and J. Sousa Ramos, Topological invariants in nonlinear boundary value problems, Chaos Solitons Fractals, 25, 65-78, 2005. 